\title{
AUTHOR INDEX (Volume 17)
}

\author{
Ahn, D. 111 \\ Ahn, I.-S. 195, 293 \\ Ahn, M. S. 277 \\ Ahn, S.-H. $\quad 45,189$ \\ Bae, D.-S. 59 \\ Bae, J. S. 73 \\ Baek, S. S. 215 \\ Bak, S.-H. 141 \\ Bak, Y.-R. 51 \\ Balakin, D. Y. 469 \\ Boo, J.-H. 121, 353 \\ Bozzolo, G. 391 \\ Byeon, J. W. 99 \\ Byun, D.-J. 307 \\ Byun, S. 271 \\ Çadırlı, E. 477 \\ Canzian, A. 391 \\ Chai, Y.-Q. 497 \\ Chang, J. 33 \\ Chang, M. H. 81 \\ Che, Q. 445 \\ Chen, G. 419 \\ Chen, K. 405 \\ Chen, X. 437 \\ Chen, Y. 463 \\ Chiriac, H. 369 \\ Cho, H. 93, 337 \\ Cho, J. Y. 207 \\ Cho, S.-J. 353 \\ Cho, T. Y. 207 \\ Choi, B. C. 73 \\ Choi, E. 111 \\ Choi, H. S. 245 \\ Choi, J.-M. 257, 307 \\ Choi, K.-H. 87 \\ Choi, S.-H. 195 \\ Choi, S.-S. 105 \\ Chu, M.-C. 59 \\ Chun, D.-M. 45, 189 \\ Chun, H. G. 207 \\ Chung, J.-K. 289 \\ Chung, T.-M. 307 \\ Chung, Y.-M. 51 \\ Cui, Y. 425
}

Do, K.-R. 195

Fan, J. 369

Fan, S. 445

Fuh, J. Y. H. 159

Gim, J. 111

Girardet, C. 431

Grado-Caffaro, M. 441

Grado-Caffaro, M. A. 441

Gu, H.-B. 15

Guo, Y. 397

Ha, N. R. 153

Ham, Y.-S. 27

Han, B. S. 173

Hassan, Z. 497

He, H. 397

He, L. 419

He, Y. 451

Hejun, L. 487

Hong, C. K. 15

Hong, J.-K. 105, 229

Hong, M. H. 383

Hu, R. L. 129

Hu, X. 463

Huang, P. 265

Hwang, K. H. 153

Hwang, M.-J. 15, 51

Hwang, T. 39

Jang, J. M. 235, 241

Jeong, E. D. 73

Jeong, G.-H. 87

Jeong, I.-S. 185

Jeong, J. H. 73

Jeong, S. H. 121

Ji, C. 463

Jia, J.-F. 437

Jiang, L. 463

Jiang, Y. 397

Jiansirisomboon, S. 1

Jin, E. M. 15

Jin, S.-E. 307

Jo, H.-H. 93

Jo, N.-J. 63

Joo, Y. K. 207
Ju, J.-H. 51

Jung, D. 353

Jung, E. J. 105

Jung, H. 111

Jung, K. 45, 189

Jung, Y. R. 73

Jung, Y.-G. 337

Kang, D.-S. 251

Kang, E. S. 215

Kang, I.-J. 165

Kang, J. H. 207

Kang, S. G. 289

Kang, T.-J. 245

Kang, W. N. 289

Kaya, H. 477

Kezhi, L. 487

Khodin, A. 283

Kim, B. 307

Kim, C. G. 307

Kim, C. J. 177,289

Kim, D. 111

Kim, D. K. 215

Kim, D. Y. 121

Kim, D.-G. 359

Kim, D.-H. 293

Kim, D.-J. 329

Kim, D.-W. 195

Kim, G. 39

Kim, G. C. 153

Kim, H. 39

Kim, I.-H. 235, 241

Kim, J. 111

Kim, J. H. 73, 105

Kim, J.-H. 229, 337

Kim, J.-J. $\quad 87$

Kim, J.-K. 201

Kim, K. B. 245

Kim, K.-J. 105

Kim, K.-S. 329

Kim, K.-W. 15

Kim, M.-H. 121

Kim, M.-K. 63

Kim, M.-S. 45

Kim, M.-Y. 311 
Kim, S.-H. 251

Kim, S.-J. 9, 69, 141, 299

Kim, S.-O. 283

Kim, T. S. 245

Kim, W.-J. 87

Kim, Y. J. 33

Kim, Y.-J. 9, 257

Ko, S.-H. 235, 241

Ko, Y.-J. 59

Ko, Y.-K. 201

Koh, J.-H. 27

Koo, B. H. 33, 271

Kumar, A. S. 383

Kumar, S. 33

Kwon, E.-P. 251

Kwon, J.-Y. 337

Kwon, S. C. 207

Lau, W. S. 497

Le, X. 463

Lee, B.-J. 87

Lee, B.-S. 93

Lee, C. G. $\quad 33,271$

Lee, C. S. $\quad 45,189$

Lee, C. W. 81

Lee, C.-W. 201

Lee, C.-H. 229

Lee, D. 307

Lee, D. B. 141

Lee, D.-B. 9

Lee, H. 39

Lee, J. C. 9, 141

Lee, J. K. 59, 153, 245, 283

Lee, J.-H. 359

Lee, M. H. 245

Lee, N. H. 289

Lee, N.-E. 121

Lee, S. 307, 353

Lee, S. J. 215

Lee, S.-C. 165

Lee, S.-M. 87, 317, 323

Lee, S.-W. 21, 165

Lee, S.-Y. 337

Lee, T.-K. 177, 289

Lee, W. 111, 235, 241

Lee, W. H. 245

Lee, Y.-J. 63

Lei, M. 463

Li, C. 419

Li, K. Y. 129

Li, M.-X. 207

Li, W.-J. 437

Li, X. 457

Li, X. P. 369

Liang, J. 463

Liao, Y. 405

Lim, C. S. 383

Lim, J. 111
Lim, S.-G. $\quad$ 185, 195, 293

Lin, Y. 383

Lim, Y. J. 177

Liu, F. $\quad 135,363$

Liu, G. 265

Liu, W. 405

Liu, Y. 457

Lu, L. 129, 159

Lu, P. 261

Ma, H. 425

Ma, S. 159

Ma, X. 437

Ma, Z. 405

Mathew, V. 111

Minamidate, Y. 147

Moon, B. K. 73

Moon, M.-S. 251

Moon, S.-H. 27

Mosca, H. O. 391

Mun, G. 39

Na, M. K. 277

Nam, S.-H. 121

Nguyen, T. D. 9

Ning, N. 369

Oh, T.-S. 311

Osovskii, V. D. 469

Paek, S. 111

Paik, U. 337

Paik, Y. 111

Pan, L. 493

Park, B.-K. 15

Park, H. Y. 277

Park, H.-W. 185

Park, I.-M. 257

Park, J.-C. 69

Park, K.-H. 15

Park, N.-K. 105, 229

Park, S. C. $\quad 177,289$

Park, S.-K. 257

Park, Y.-H. 257

Peng, C. 419

Petrova, N. V. 469

Pham, H. C. 329

Politano, A. 411

Ptushinskii, Y. G. 469

Rahman, M. 383

Ramseyer, C. 431

Rhee, C. K. 173

Rong, C. 463

Ryu, K.-S. 51

Said, M. 431

Şahin, M. 477

Sato, T. 147

Saw, K. G. 497
Seo, D. S. 153

Seo, D. Y. 141

Seo, Y. J. 33

Shanpeng, S. 375

Shim, S.-Y. 185, 293

Shin, E.-C. 87

Shin, J.-S. 245

Shin, S.-G. 241

Shin, Y.-S. 201

Shon, I.-J. 251

Shuping, L. 487

Soh, A. K. 129

Son, S.-H. 235

Song, G.-A. 245

Song, J. I. 271

Song, K. J. 289

Song, W. 45, 189

Su, F. 493

Subin, H. 375

Sun, Y.-J. 437

Sun, Z. 493

Sung, Y.-R. 293

Tam, P. L. 129

Tan, L. S. 383

Thongsanitgarn, P. 1

Ting, C.-J. 345

Tneh, S. S. 497

Tsai, H.-Y. 345

Uhm, Y. R. 173

Wang, C. 265

Wang, G. 437

Wang, K. 271

Wang, L. 265, 425

Wang, Q. 457

Wang, S. 397

Wang, X. 419

Wang, Y. Q. 271

Wang, Z. 265, 463

Watcharapasorn, A. 1

Woo, K.-D. 251

Woo, Y.-B. 299

Wu, J. 369, 405

Wu, L. 405

Xin, J. 463

Xiuyan, H. 375

$\mathrm{Xu}$, J. $\quad 405,463$

$\mathrm{Xu}, \mathrm{L} . \quad 405$

Xue, D. 135, 261, 363

Xue, Q.-K. 437

Yakovkin, I. N. 469

Yam, F. K. 497

Yanfeng, L. 375

Yang, D. 21

Yang, F. 405

Yang, G. 457 
Yang, H. K. 73

Yang, L. 419, 425

Yang, Z. X. 153

Yeo, J.-C. 45

Yeom, J.-T. $\quad 105,229$

Yi, D.-K. 337

Yin, S. 147

Yoo, D. G. 121

Yoo, S. 201

Yoon, J. H. 207

Yoon, J.-S. 223
Yu, X. 457

Yuefei, Y. 375

Yun, J.-J. 15

Zhang, F. 445

Zhang, J. 457

Zhang, L. 463

Zhang, P. 463

Zhang, S. H. 207

Zhang, T. 437

Zhang, X. 463
Zhang, Y. 463

Zhang, Y. F. 159

Zhao, S. 425

Zheng, S. 265

Zheng, Y. 493

Zhi, W. 451

Zhijia, Y. 375

Zhou, B. O. 451

Zhou, W. 457

Zhu, X.-G. 437

Zorgati, H. 431 